\title{
A New Mathematical Approach for Pattern Generation
}

\author{
Navdeep Kumar \\ Abraham Consultancy Services Pvt. Ltd. India
}

\author{
Pankaj Garg \\ Madurai Kamaraj University, India
}

\begin{abstract}
Actual CAD systems are still monolithic and are based on complex geometrical models. Our aim is to implement a new mathematical approach based on fractal geometry integrating the advantage of web technology and providing a novice end-user with an easy- to-use system. In order to get the same performances as a local system, it is necessary to provide our system with adequate data representation aid architecture. This paper presents a framework of web-based system to generate the patterns for ornamental objects. The emphasis of this paper is to outline of methodology and architecture to develop the system, which depends on classifying the elements influenced in the conceptual design stage. The proposed system provides as tool for customer to generate design alternatives according to designer's preferences. Thus the customer can directly explore more alternatives and generate designs with his/her styles from predefined images in the data base of system. Accordingly, to the requirement of customer, the whole system regenerate the designs using the power of macro programming. This whole system will use the power of Web technology and macro programming to generalize the designs.
\end{abstract}

Keywords: Fractal geometry, Self-similar patterns CAD, Fractal Curves, Macros.

\section{INTRODUCTION}

Fractal geometry is, like all other forms of geometry, the mathematics of size, shape and special relationships. The point where it differs from the Euclidean geometry is that it deals with shapes that are infinitely irregular. As Euclidean geometry is the natural way of rep-resenting man-made objects, fractal geometry is the natural way of representing objects that occur in nature [1]. In addition, many products in jewellery industries have a similar natural design. Computer-aided design (CAD) is at the heart of the present industrial revolution. The design capabilities of contemporary CAD systems are either based on the solid modelling method or on the surface modelling method. The surface modelling method gives a precise and a convenient way to sculpture free-form surfaces, while the solid modelling method provides an unambiguous and information-complete solution to define a three-dimensional object [2]. On the other hand, rapid prototyping (RP) technology is used with CAD to make available the physical generation of a solid object. Natural objects, such as mountains, clouds, and trees have irregular or fragmented features with self-similarity. These natural objects can be realistically described by fractal geometry while Euclidean geometry is mainly used to represent man-made objects such as squares, circles and triangles. It is thus valuable to develop a method that can manufacture self-similar designed objects. Here, fractal geometry is used in the representation since it has the important geometric property of self-similarity. Fractal geometry can also provide a simple way to represent jewellery design which needs to be aesthetically appealing to humans. However, no commercial CAD systems are to the efficient manipulation of fractal geometry. For example, the non-uniform rational B-spline (NURBS) method in surface modelling cannot be used to model a fractal curve due to fragmentation. Further, the constructive solid geometry (CSG) method in solid modelling will represent an object hierarchically, but it cannot model the self-similarity in the fractal object. [3] Therefore, it is valuable to apply fractal geometry integrating with web technology for customized pattern designs for aesthetic objects.

\section{THE FRACTAL GEOMETRY}

The word "fractal" was coined less than twenty years ago by one of history's most creative mathematicians, Benoit Mandelbrot, whose seminal work, The Fractal Geometry of Nature, first introduced and explained concepts underlying this new vision. A Fractal is generally "a rough or fragmented geometric shape that can be split into parts, each of which is (at least approximately) a reduced-size copy of the whole," a property called selfsimilarity. A mathematical fractal is based on an equation that undergoes iteration, a form of feedback based on recursion. This definition covers several geometries that are self-similar under several scales. Two main types of fractals are more popular d including iterative function system (IFS) fractals (e.g., selfintersecting fractal objects, simply connected fractal objects, multiply connected fractal objects, natural objects, area filling fractal objects, etc.) and complex fractals (e.g., quaternion Mandelbrot set,quaternion Julia set, filled-in Julia set, contour Julia set, dust Julia set, Dendrite Julia set, etc.) Barnsley and Demko [4] first propose the iterative algorithm method called IFS. Minkowski, Hilbert, Peano curves, Sierpinski gasket, the Sierpinski carpet and the Koch Island are typical examples that could be found in the literature of fractals. All these intricate geometries are built using an IFS method which can be expressed mathematically with Eq.(1), Eq.( 2 )and Eq.( 3).

$$
\mathrm{A}_{\mathrm{i}}=\mathrm{W}\left[\mathrm{A}_{\mathrm{i}-1}\right] \quad \mathrm{i}=0,1, \ldots \ldots \ldots, \infty-----(1)
$$

Where $W$ is the Hutchinson's geometrical transformation Algorithm 
$W[A]=w_{1}(A) \bigcup w_{2}(A) \bigcup \ldots \ldots . . w_{n}(A)-----(2)$

and $w$ being an affine transformation. A recursive affine transformation is

$$
\left(\begin{array}{l}
x_{n+1} \\
y_{n+1}
\end{array}\right)=\left(\begin{array}{ll}
p & q \\
r & s
\end{array}\right)\left(\begin{array}{l}
x_{n} \\
y_{n}
\end{array}\right)+\left(\begin{array}{l}
u \\
v
\end{array}\right)----(3)
$$

After an infinite number of iterations, the initial set $A o$ or initiator, which started the iterative algorithm, is transformed into the set $A$ that is the fractal. Each intermediate state $A i$ is called prefractal. The complex fractals are generated and represented by complex set mappings and picture elements (pixels) respectively. For example, B. Mandelbrot (1924) a brilliant Polish mathematician, had developed and popularized a relatively novel class of mathematical functions know as Mandelbrot set. The function is defined by a simple recurrence formulation in the complex plane [5], mathematically as shown in equation 4 ,

$$
\mathrm{Z}_{\mathrm{n}}=\mathrm{Z}_{\mathrm{n}-1}^{2}+\mathrm{C}
$$

\section{LITERATURE REVIEW}

Very little research for manufacturing fractal objects has been reported in the literature. Kerekes [6] is probably the first to generate a fractal tree structure in three dimensions with Stereolithography (SL) RP machine. The example is generated without any help from CAD as the structure is extremely complex. However, Kerekes has not reported much detail. Lee et al [7] has proposed an integrated CAD and RP approach. The discussion is only based on Euclidean geometry domain. In fact, CAD and RP systems available in the market for jewelry making cannot handle fractal geometry yet.

Soo and Yu mentioned a method for the Rapid Prototyping (RP) of the fractal geometry objects. A workflow for rapid prototyping fractal geometry has been outlined $[8,9]$.

A mechanism for performing recursive shape computations is proposed based on the concept of shape grammar. A general paradigm has been developed for a computer implementation supporting this computation in the algebras of points and lines in two-dimensional situation [10-11].

Vishal Gulati [12] proposed a methodology to automate the arena of design and manufacturing for producing ornamental products of various engraved forms.

All these researchers proposed the integration of CAD with manufacturing integrating Fractal techniques. But there is lack of customization of designs from the customer perspective.

Therefore, in this research, an integrating system is proposed, which is based on fractal geometry and information technology. The tool is used to link computer aided design (CAD) system and customers and designers

\section{BASIC TYPES OF FRACTAL CURVES}

The Snowflake, Hilbert, Peano, and Gosper are mathematical curves found in the literature of self similar fractals [11, 13]. Each of the fractal curves is defined by a modification rule, sub dividing into smaller pieces so that rule can be applied recursively to modify each piece in the same manner. These curves are generated by recursive processes.

\subsection{Koch Curve fractal}

The Koch Curve fractal is one of the most popular and well known fractals. The Koch Curve fractal is interesting because it is similar to some fractals found in nature. In some ways, the Koch fractal looks like the coastline of a continent or an island. The construction of the Koch curve goes like this: Start with a line segment, say $[0,1]$, and replace the middle third subsequent $[1 / 3$, $2 / 3$ ] with the other two side of the equilateral triangle constructed on it, giving a zig-zag line consisting of four segments of lengths $1 / 3$. Now the same construction is repealed (going "to the outside") on each of these four segments, then on each of the sixteen resulting segments, and so on. Figure 1 shows the first five stages of the construction.

\subsection{The Peano Fractal Curve}

The Peano Curve (also known as the Hilbert plane-filling curve) is a subset of the family known as space-filling curves. These curves just so happen to also be fractals. Generally a space-filling curve is defined as a curve that can pass through every point of a finite region of an $n$-dimensional space. In this case, $n$ must be greater than or equal to 2. For instance, if $n$ is equal to 2 , then the space-filling curve must pass through every point of the square without leaving any empty spaces. Few stages of the Peano Curve are shown in figure 2.An initial shape is drawn in a unit square and shrinked to half its size and at the same time the current plane is divided into a grid of four equal sizes. Then placing of one of four copies of this new curve into each new grid segment is done. The top two copies are placed in the same orientation as the original curve. The bottom two copies, on the other hand, are rotated a quarter turn, the left one clockwise and the right one counter-clockwise. Finally, ends of the four copies is connected so that they form one continuous curve. The resulting image is the second stage of the Peano Curve. This algorithm is then repeated again for each of the four clones. This would yield the third state of the Peano Curve. Eventually, after $n$ iterations, we arrive at the true representation of the Peano Curve. The image below shows the first five stages of the Peano Curve. 


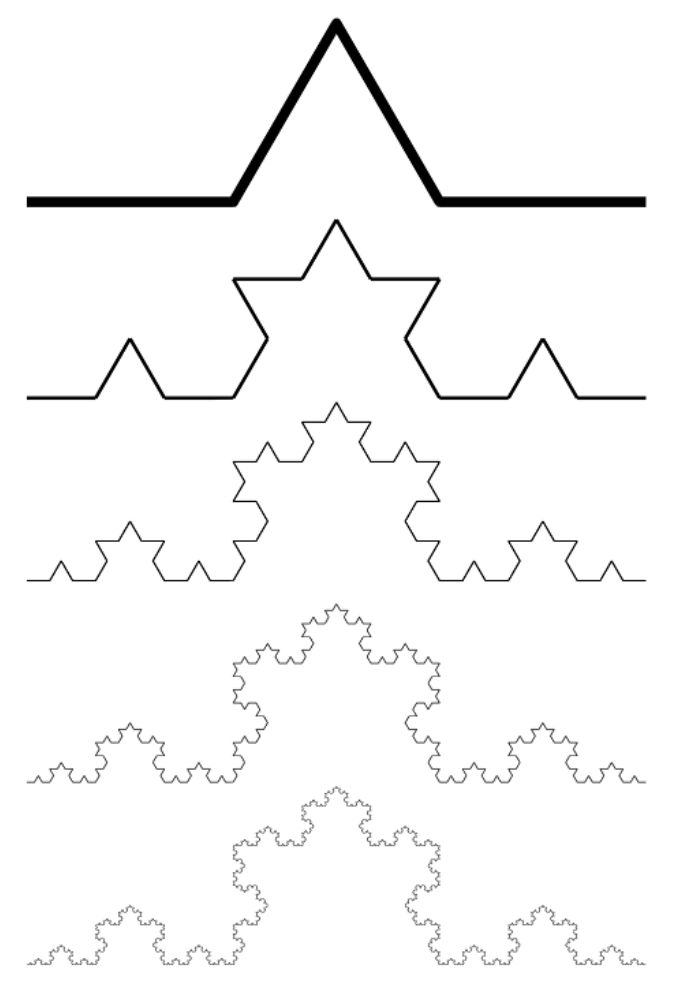

Figure 1: Five Successive stages of Koch Curve

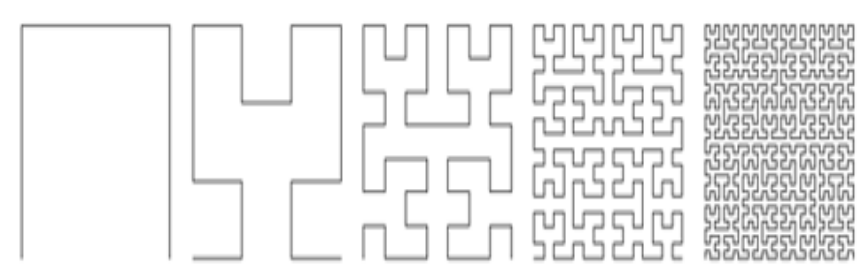

Figure 2: Successive stages of Peano Curve [14]

\subsection{Koch Snowflake Fractal Curve}

The Koch snowflake is a mathematical curve and one of the earliest fractal curves. It is based on the Koch curve. The Koch snowflake can be constructed by starting with an equilateral triangle, then recursively altering each line segment as follows:

Start with an equilateral triangle T. Scale $T$ by a factor of $1 / 3$ and place 3 copies along each of the three sides of $\mathrm{T}$ as illustrated in the figure 3 to form a new image $S(1)$. Next scale $\mathrm{T}$ by a factor of $1 / 9$ $=(1 / 3)^{\wedge} 2$ and place $12=4 * 3$ copies along the sides of $\mathrm{T}(1)$ as illustrated to form the image $S(2)$. For the next iteration, take $48=4 * 12$ copies of $T$ scaled by a factor of $1 / 27=(1 / 3)^{\wedge} 3$ and place them around the sides of $S(2)$ to form the image $S(3)$. Continue this construction. The Koch Snowflake is the limiting image of the construction.

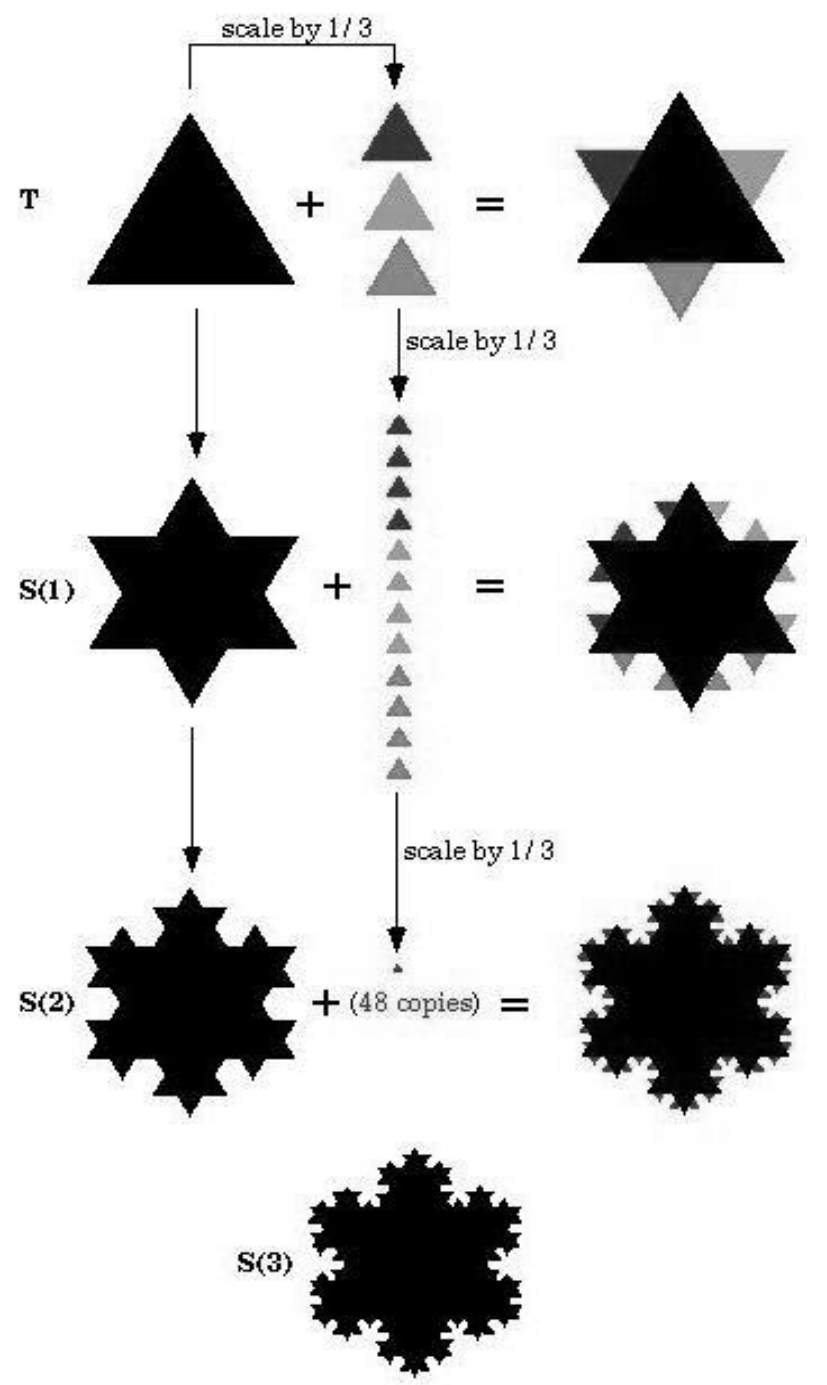

Figure 3: Successive Stages of Koch Snowflake Curve [15]

\subsection{The Hilbert Fractal Curve}

A Hilbert curve is a continuous fractal space-filling curve first described by the German mathematician David Hilbert.The Hilbert curve is a more complicated construction. The easiest definition is grammatical, using pairs of elements. The members of each pair are geometrically identical, but in the replacement rules, they're replaced differently.

- $\mathrm{Right}_{1}$, and Right 2 : a clockwise right angle with legs length $\mathrm{L}$.

- Left $_{1}$ and Left $_{2}$ : a counterclockwise right angle with legs length $\mathrm{L}$.

- Straight $_{1}$ and $S_{\text {traight }}$ : a straight line segment with length $2 \mathrm{~L}$. 
The replacement rules are:

$$
\begin{array}{ll}
\text { 1. } & \text { Left }_{1} \rightarrow \text { Straight }_{2}, \text { Left }_{1}, \text { Left }_{2}, \text { Right }_{1} \\
\text { 2. } & \text { Left }_{2} \rightarrow \text { Right }_{2}, \text { Left }_{1}, \text { Left }_{2}, \text { Straight }_{2} \\
\text { 3. } & \text { Right }_{1} \rightarrow \text { Left }_{1}, \text { Right }_{2}, \text { Right }_{1}, \text { Straight }_{1} \text {. } \\
\text { 4. } & \text { Right }_{2} \rightarrow \text { Straight }_{1}, \text { Right }_{2}, \text { Right }_{1}, \text { Left }_{2} \text {. } \\
\text { 5. } & \text { Straight }_{1} \rightarrow \text { Right }_{2}, \text { Left }_{1}, \text { Left }_{2}, \text { Right }_{1} \\
\text { 6. } & \text { Straight }_{2} \rightarrow \text { Left }_{1}, \text { Right }_{2}, \text { Right }_{1}, \text { Left }_{2}
\end{array}
$$

Below is a series of stages of the Hilbert curve, using a square (1, Left $_{2}$, Left $_{1}$, Left $_{2)}$ as an initiator, and the above rules as the generator. The distortions in the far right stage are the result of a minor glitch in my turtle graphics package, not a real property of the curve.)

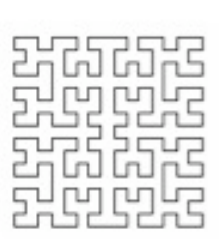

2 iterations

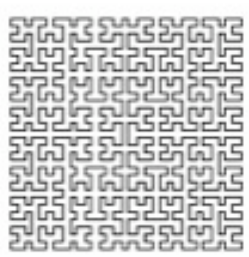

3 iterations

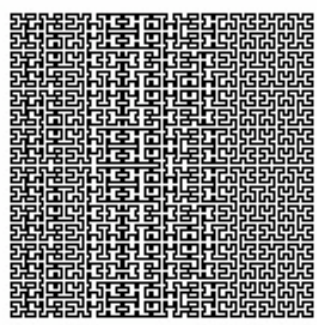

4 iterations
Figure 4: Successive Stages of Hilbert Curve [16]

\section{IMPLEMENTATION}

The architecture of proposed integrated system is shown in figure 5. It composes two main components that are CAD program and database working on TCP/IP protocol. Then user can easily access to it by using WWW browser via the Internet. The Internet CAD system is open for any client who wants to select a particular pattern design for an order. One of the purpose of the system, the fractal theory for pattern design will be combined with the system by using the CAD program. Furthermore, the database of the system includes concept, know-how, method of calculation, and many images. The system should provide interactive communication of pattern design between the system and its user as designer by using the inference engine.

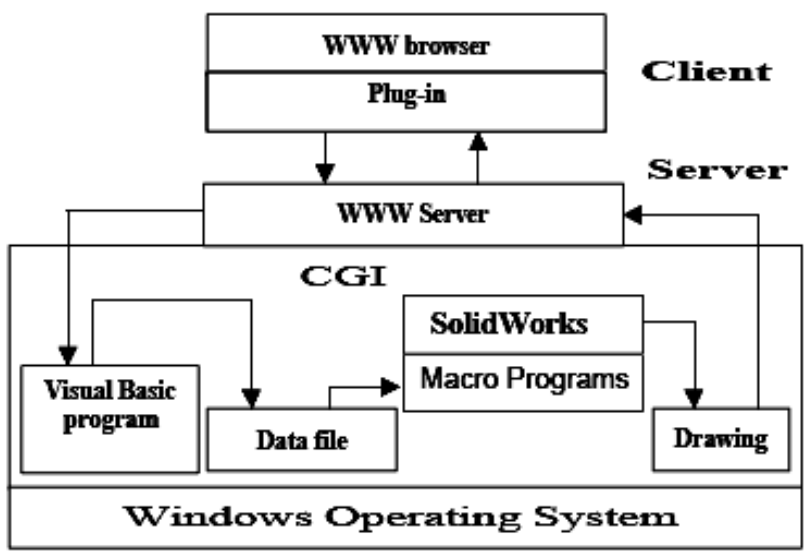

Figure 5: Architecture of Web-based pattern design system
Adding the Internet in the system to some communication method at the customer level. In this research work an algorithm for Koch curve is programmed in Visual $\mathrm{C}++$ environment. Different patterns are generated using Koch curve fractal technique.

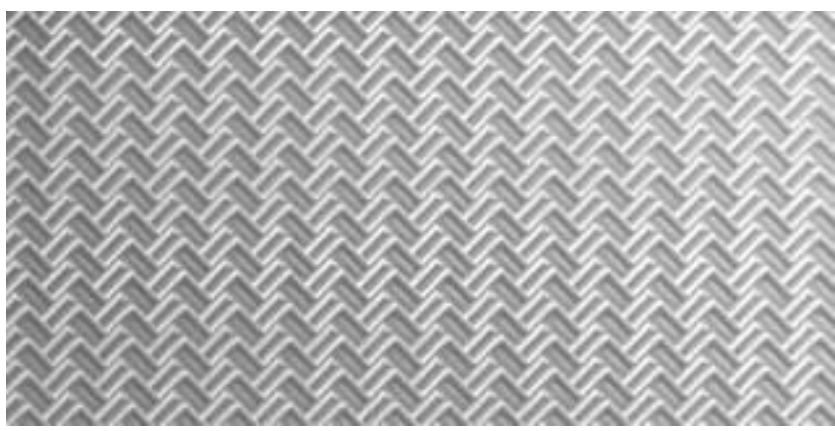

Figure 6 : Self-similar Patterns Engraved on Surface

Some of these patterns are projected on a free-form surface for engraving or embossing. Tool paths are generated using CNC milling to engrave patterns on the surface of wood. One of the selfsimilar fractals is engraved on the surface using CNC Milling system as shown in figure 6 .

\section{CONCLUSION}

The developed system integrates the power of fractal geometry and web technology. The proposed system handovers the designing power to client directly. Thus it provides greater flexibility and customization to generate wonderful irregular shape patterns to engrave any ornamental parts such as pendants \& bracelets. To further extend the geometric coverage, fractal geometry can also be integrated with Euclidean CAD.

\section{REFERENCES}

[1] D. Hearn and M. P. Baker, Computer Graphics, Prentice Ha 2009.

[2] I. Zeid, CAD/CAM Theory and Practice, McGraw-Hill, New York ,2010.

[3] Soo S. C. and Yu K.M., 'Rapid Prototyping for Self Similarity Design', Journal of Materials Processing Technology, Elsevier, Vol. 139, pp. 219-225, 2003.

[4] M.F Barnsley and S.Demko : Proc. Roy. Soc.London p.243, 1985.

[5] B. Mandelbrot: The Fractal Geometry of nature, 1983.

[6] T.Kerekes, "Stereolithography builds computer-generated models of natural forms", Rapid Prototyping Report, CAD/CAM Publishing, Vol.2, No.4, p.1, 1992.

[7] H.B. Lee, M.S.H Ko, R.K.L. Gay et al., "Using computerbased tools and technology to improve jewellery design and manufacturing", International Journal of Computer Applications in Technology, Vol.5, No. 1, pp.72-80, 1992.

[8] Kerekes T., Stereolithography Builds Computer-Generated Models of Natural Forms, Rapid Prototyping Report, CAD/CAM Publishing, Vol. 2, No. 4, pp. 1, 1992 
[9] Soo S. C. and Yu K.M.,Rapid Prototyping for Self Similarity Design, Ninth International Manufacturing Conference, China, Vol. II, pp. 93-94, 2000.

[10] Gips J.,Shape Grammar and Their Uses, Birkhauser Verleg, Basel Und Stuttgart, 1975.

[11] Stiny G., Introduction to Shape and Shape Grammars, Environment and Planning-B, Vol. 7, pp. 343-351, 1980.

[12] Vishal Gulati, Hari Singh and Puneet Tandon , 'A CAD Paradigm to Produce Engraved Form of Ornamental Products',
National Conference on Advances in Computer networks \& Information Technology, India, II, pp. 5-9, 2009.

[13] Soo S. C. and Yu K. M., 'Tool Path Generation for Fractal Curve Making, The International Journal of Advanced Manufacturing Technology, Springer-Verlag Landon Ltd, Vol. 19, No. 1, pp. 32-48, 2002.

[14] Agnes Scott, available at http://ecademy.agnesscott.edu.

[15] ScienceBlogs, available at http://scienceblogs.com 